\title{
A GLIMPSE FROM THE INSIDE OF A SPACE SUIT: WHAT IS IT REALLY LIKE TO TRAIN FOR AN EVA?
}

\author{
Matthew A. Gast \\ United Space Alliance, LLC \\ 600 Gemini, Houston TX, 77058-2783; USA \\ matthew.gast-1@nasa.gov \\ Sandra K. Moore, Ph.D. \\ United Space Alliance, LLC \\ 600 Gemini, Houston TX, 77058-2783 ; USA \\ sandra.k.moore@nasa.gov
}

\begin{abstract}
The beauty of the view from the office of a spacewalking astronaut gives the impression of simplicity, but few beyond the astronauts, and those who train them, know what it really takes to get there. Extravehicular Activity (EVA) training is an intense process that utilizes NASA's Neutral Buoyancy Laboratory (NBL) to develop a very specific skill set needed to safely construct and maintain the orbiting International Space Station. To qualify for flight assignments, astronauts must demonstrate the ability to work safely and efficiently in the physically demanding environment of the spacesuit, possess an acute ability to resolve unforeseen problems, and implement proper tool protocols to ensure no tools will be lost in space. Through the insights and the lessons learned by actual EVA astronauts and EVA instructors, this paper will take you on a journey through an astronaut's earliest experiences working in the spacesuit, termed the Extravehicular Mobility Unit (EMU), in the underwater training environment of the NBL. This work details an actual Suit Qualification NBL training event, outlines the numerous challenges the astronauts face throughout their initial training, and the various ways they adapt their own abilities to overcome them. The goal of this paper is to give everyone a small glimpse into what it is really like to work in a spacesuit.
\end{abstract}

Copyright (C 2009 by United Space Alliance, LLC.

\section{Introduction}

The successful completion of STS128/17A, landing at Edwards Air Force Base in California on Friday, September 11, 2009, marks the end of the $30^{\text {th }}$ Space Shuttle mission in support of the construction of the International Space Station (ISS). STS-128/17A performed three EVAs; the mission's most significant task was the removal and replacement of one of two Ammonia Tank Assemblies (ATA), the supply tank for the External Active Thermal Control System (EATCS) and the most massive Orbital Replacement Unit (ORU) ever handled by astronauts in orbit. Weighed preflight at the Kennedy Space Center (KSC) as part of the preparations for the mission, the fully-loaded ATA was found to be $1850 \mathrm{lbs}$, making it a formidable object to control in the microgravity vacuum of space. To better understand the translational and rotational characteristics of the ATA, American astronauts Dr. John "Danny" Olivas and Nicole Stott, and European Space Agency (ESA) astronaut Dr. Christer Fuglesang, trained for 12 months prior to flight, using every EVA training resource available (including sessions in NASA's Virtual Reality and Neutral Buoyancy Laboratories). Through this process, the crew, together with their EVA Task instructors, developed techniques to safely manipulate the ATA during removal and replacement, culminating in the successful change-out of the Pl truss segment ammonia tank, choreographed over the first two EVAs of the STS-128/17A mission.

The success of this mission, however, was not solely dependent on the flight-specific training over the previous 12 months. In reality, flight-specific training is primarily meant to focus on two areas. First, it develops and refines 
the methods for completing the specific tasks assigned to the mission, and second, it choreographs these individual tasks into an efficient timeline. EVA time is an expensive commodity, and efficiency is a premium that demands constant attention throughout the flightspecific training flow. But the fundamental skills of the assigned crewmembers play as much a part in the efficiency of the timeline as the composition of the tasks themselves.

For this reason, there is significant emphasis placed on generic training. A newly selected Astronaut Candidate (ASCAN) undergoes rigorous training, broken into two categories: basic generic training, referred to as the ASCAN Program, and advanced training, termed the Skills Program. Through the progressive philosophy upon which these programs are designed, ASCANs attain a strong foundation of the elementary skills that are essential to success in the EVA environment.

As one might expect, the programs focus in part on the technical aspects of EVA (i.e. improving mechanical aptitude, developing the ability to proficiently maneuver the Extravehicular Mobility Unit (EMU)). What some might find surprising, however, is the level of emphasis placed on the cognitive aspects of EVA. Dr. David Wolf, an astronaut veteran of six ISS-construction spacewalks, including most recently three on STS-127/2JA, flown in July of 2009 , often refers to the cognitive aspects as "putting on your EVA brain." By this, Wolf implies that, much like a chess match, EVA success relies heavily on thinking ahead and knowing the proper moves. Since EVA requires both a physical and mental aptitude, the ASCAN and Skills Programs must address both in a way that equally nurtures the talents and improves the deficiencies of each student, all the while maintaining an acceptable standard of qualification for all.

As could be imagined, the journey an ASCAN must go through to attain EVA qualification is not a trivial one. Expectations are high and each student is challenged on a continuous basis. The programs maintain a level of flexibility that allows the EVA Task instructors to modify training as required to further emphasize strengths, and to exploit individual weaknesses in an attempt to surmount those shortcomings, be they physical, mental or a combination of both.

The journey, they say, begins with the first step. For an ASCAN delving into the world of EVA, that journey's beginning is marked with his or her first foray into the Neutral Buoyancy Laboratory, in the first of the four ASCAN Program training sessions, referred to as Suit Qualification.

This paper begins by examining the importance of skills-based generic training in general. It then continues with the detailed discussion of the numerous aspects that encompass the Suit Qualification training event, from the body measurements taken to properly fit the EMU, to the basic evaluation criteria used to assess each student. Through the accounts of astronauts who have taken this journey, this paper will offer a glimpse into the initial training that lays the foundation upon which the success of STS-128/17A - and the other 101 U.S.-based EVAs in support of ISS construction - is built.

\section{The Scope of ISS Construction}

The International Space Station has been in orbit since STS-88/2A mated the first U.S. module, the Node 1 Unity module, to the first Russian segment, the Functional Cargo Block (FGB) Zarya (Sunrise) module, in December of 1998. Astronauts Jerry Ross and James Newman conducted three EVAs totaling 21 hours, 22 minutes in duration, to connect the two modules' cabling and connectors, setting the cornerstone of the first international laboratory in space.

Since that time, the effort to assemble the ISS has been monumental. Spacewalks have been conducted during joint missions (i.e. while the space shuttle is docked to ISS), as well as during steady-state Increment operations (i.e. long duration spaceflight on the ISS) using both the EMU and the Russian ORLAN spacesuit. NASA is joined by the Russian Space Agency (RSA), the European Space Agency (ESA), the Canadian Space Agency (CSA) and the Japanese Aerospace Exploration Agency (JAXA) in providing astronauts who have helped assemble ISS thus far.

The following are some of the numbers associated with the construction of the ISS through STS-128/17A. (Note: At the time of publication, six missions still remain on the manifest to complete all assembly and outfitting)

- 133: Number of ISS-Construction EVAs

- 90: Joint Shuttle/ISS EVAs

- 29: Increment ORLAN EVAs

o 14: Increment EMU EVAs 
- 91: Number of astronauts who performed least one EVA in support of ISS construction
- 65: NASA Astronauts
- 18: RSA Cosmonauts
- 4: ESA Astronauts
- 3: CSA Astronauts
- 1: JAXA Astronaut

- 830 hours, 51 minutes: Time spent EVA in support of ISS construction

- $84 \%$ : Percentage of ISS assembly complete

- 710,966: mass in orbit of ISS (in lbs)

(Wedlake, 2009 and NASA PAO STS-128 / 17A by the numbers, 2009)

In the past 11 years representatives from seven separate countries have been directly involved in the assembly of the ISS in orbit, overcoming language barriers, technical problems, launch slips and, at times, in-situ preparation, to grow the ISS and keep it functional.

A considerable portion of the success can be attributed to the flight-specific training gained in the months leading up to a mission. Some of the most spectacular successes, however, have come from the challenges presented by real-time contingencies that were not anticipated. The ability to overcome such contingencies highlights the profound importance of generic, skills-based training.

\section{A Solar Array Gets a Set of Cufflinks}

Nowhere is the importance of generic, skills-based training more evident than the challenge that befell the crew of STS-120/10A. The mission was given two primary EVA objectives. First, deliver the Node 2 Harmony module to the ISS, and second, relocate the P6 truss segment to the port side of the vehicle and redeploy its solar arrays. It was this second task that produced one of the greatest challenges of ISS construction.

It was known from STS-116/12A.1 that during preparation activities for truss segment relocation, the power channel $2 \mathrm{~B}$ solar array of the P6 truss had difficulty retracting. And while the STS-120/10A crew had conducted generic solar array contingency training preflight, the damage discovered during redeployment was unlike anything that had been expected. Two sections of the array had begun to separate, and deployment was halted over the concern that the array would continue to separate, essentially "unzipping" into two nonfunctional pieces.
The team on the ground at NASA's Mission Control Center spent the next 48 hours developing a plan to repair the damaged array, including using only hardware already onboard the ISS and space shuttle to construct a "bandage" that could hold the damaged section of the solar array together. In the end, the ground team uplinked a procedure to the ISS, directing the crew to construct a set of "cufflinks" for the solar array. The job of installing the cufflinks then fell to veteran spacewalker Scott Parazynski and first-time flyer Doug Wheelock.

Using the Orbiter Boom as an extension, the Space Station Robotic Manipulator System (SSRMS) maneuvered Parazynski to the damaged array, stretching nearly to its full extent. Then, relying on his vast experience (Parazynski was a veteran of six prior spacewalks) and his skills-based training, Parazynski systematically installed three cufflinks (Figure 1). Upon completion, the solar array was successfully extended to its nominal operating position. That array continues to provide the ISS with power generation capabilities.

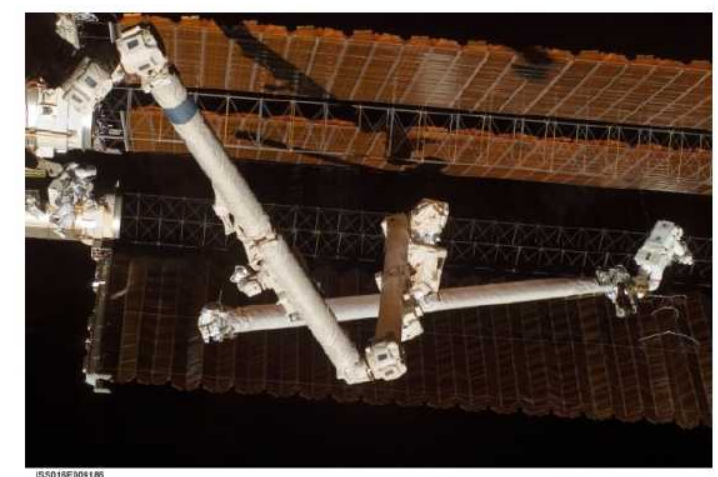

Figure 1. Astronaut Scott Parazynski maneuvering away from the damaged solar array. Three cufflinks, visible just below his right knee, hold the damaged region of the solar array together.

Source: iss016E009186.jpg (photo added 11/03/2009)

\section{The Purpose of Generic, Skills-Based Training}

The repair of the power channel 2B solar array during STS-120/10A appears simple enough: stand atop the SSRMS, insert three cufflinks into the solar array and back away. Add in the shock hazard associated with a solar array that sees 45 minutes of sunlight every 90 minutes, however, and things become more challenging. Combine the fact that the SSRMS is a dynamic entity, tending to oscillate under 
any imparted load, and the task becomes daunting. To further obfuscate the task, Parazynski had no preflight training to understand the worksite or the dynamics of working atop the SSRMS/Orbiter Boom stackup. Knowledge and confidence in Parazynski's experience and fundamental EVA skills allowed mission designers to develop an EVA procedure they believed could be performed both safely and successfully.

Due to the shock hazard, Parazynski was directed to avoid any physical contact between the array and the EMU; he was to touch the array only with the cufflinks, and with tools wrapped in non-conductive Kapton tape. Yet, even with the myriad challenges set before him, Parazynski was still successful; his experience was able to fill in the gaps that existed due to the lack of task-specific training.

However, what if it had not been Scott Parazynski, a seasoned spacewalker, onboard the ISS during this contingency? What if it had been a rookie astronaut who was asked to perform this task? The reality is that the outcome would have likely been the same. The nuances of completing the repair may have been different, but the skillsbased generic training each EVA-qualified crewmember receives would have been sufficient to prepare him or her for a task of this complexity.

A flight-specific training flow for a joint EVA (an EVA conducted while the space shuttle is docked to the ISS) uses a training ratio on the order of $5: 1$ to $7: 1$, meaning that for each EVA conducted on orbit, the assigned crewmembers will practice that EVA five to seven times on the ground. The training arena of choice is NASA's Neutral Buoyancy Laboratory, housed at the Johnson Space Center (JSC) in Houston, Texas. The NBL pool is $202 \mathrm{ft}$ in length, $102 \mathrm{ft}$ in width, $40 \mathrm{ft}$ in depth, and holds 6.2 million gallons of water, and since 1995, it has been utilized for crew training and for EVA procedure and hardware development. Each $\mathrm{NBL}$ training event (often referred to colloquially as "a run") is six hours in duration. So for any given EVA, each assigned crewmember will spend 30 to 42 hours underwater preparing. While this seems like a considerable amount of time, it must be qualified.

The training flow for the ASCAN program is comprised of four NBL runs. The training flow for the Skills Program is comprised of an additional five to 10 runs. Thus, an ASCAN spends up to 14 separate sessions underwater on the way to EVA qualification. While this is not significantly greater than training for a single EVA, consider this: of the 73 U.S-trained EVA astronauts who have performed an ISS EVA, 16 of them, or $\sim 22 \%$, have conducted only one. An additional 10 astronauts have conducted only two ISS EVAs. In all, more than one-third $(-36 \%)$ of all U.S.trained EVA astronauts to perform a spacewalk in support of ISS construction have spent, at most, 14 separate sessions in the NBL preparing for their flights.

And at any time, during any mission, the EVA crewmembers could have been faced with a contingency as challenging, or in some cases more challenging, than the one that faced Parazynski and Wheelock during STS-120/10A. For this group of astronauts, however, gaps in their knowledge could not have been filled by the vast experience gained during previous missions. For these astronauts, the primary asset they would need to rely upon was the skillsbased generic training they received on their way to EVA qualification.

Experience is priceless, but if the construction of the ISS was relegated only to experienced EVA crewmembers, construction would have progressed at a very slow rate. The ISS has demanded 133 EVAs over the last 11 years; had these been conducted only by experienced crewmembers, the demands placed upon each crewmember would have significantly affected the quality and quantity of the flightspecific training.

The purpose of generic, skills-based training is to take the experiences of the veteran astronauts and pass it along; by doing so, the capabilities of the world's spacewalking community are broadened. This gives NASA and its international partners a greater base of talent, ensuring that the crewmembers who will inhabit the ISS for years to come are capable of maintaining it as a world-class, fully functional laboratory in space. That training begins with the Suit Qualification.

\section{Suit Qualification: What Is It?}

The Suit Qualification run in the NBL is the first of four NBL runs in the ASCAN Program. More specifically, it is an astronaut's first EMU training session in the underwater environment of the NBL. Every NBL run, with few exceptions, involves a two-person EVA team. One crewmember is designated EV1, the team leader, while the other is designated EV2, 
the complementary spacewalker. This assignment of roles and responsibilities becomes more significant as training progresses, but at the point of the Suit Qualification, it is merely semantics.

The Suit Qualification is designed as a relatively low intensity introduction to working in the EMU. As stated earlier, a typical NBL run is six hours in duration. To maintain the Suit Qualification's low intensity, however, this first venture in the EMU is limited to three hours in duration. This is done for a number of reasons: to limit exhaustion, to emphasize the importance of learning basic skills without succumbing to sensory overload, and to avoid physical injury due to a less-than-optimal suit fit. It is not as simple as showing up at the NBL the day of the Suit Qualification and checking the box. First, each ASCAN must complete three prerequisites.

\section{Suit Qualification: The Prerequisites}

\section{Suit Fit}

The EMU is, in essence, a selfcontained spacecraft, and as such, it is a spacewalking astronaut's only protection from the harshness of space. As students quickly learn, however, it can also be the most formidable of foes. The mantra taught is "don't fight the suit."

The pressure inside the EMU in the $\mathrm{NBL}$ is maintained at 4.0 pounds per square inch delta (4.0 psi higher than its surroundings, regardless of depth). The EMU is comprised of a number of modular components, including a Hard Upper Torso (HUT), a Lower Torso Assembly (LTA), lower and upper arm assemblies, boots and gloves. The LTA, arm assemblies, boots and gloves are all referred to as soft goods, meaning they are made of pliable materials. The HUT, on the other hand, is almost like an exoskeleton, rigid and unyielding. The soft goods components are therefore directly affected by changes in internal pressure. One of the most surprising discoveries when first experiencing full pressurization is the rigidity of the soft goods components. "It almost feels as though you are encased in wet concrete, and as the suit is pressurized, it feels like the concrete hardens," one astronaut described. With this increased stiffness once pressurized, one can imagine that its fit is of utmost importance.

The modularity of the EMU allows it to accommodate a wide range of body shapes and sizes, but it also means that the majority of possible configurations are not optimal for any one body type. To optimize the suit fit for each ASCAN, he or she goes through three separate fittings, often referred to as "fit checks," each two hours in duration. The first fit check, performed in the Suit Laboratory within the NBL facility, simply takes all relevant anthropometric measurements. Stature, chest circumference, inter-wrist distance, vertical trunk diameter, thigh deltoid breadth and head length are but a few of the 38 separate measurements taken to calculate the proper sizing of each modular component of the EMU. Maybe the most intimidating aspect of this first fit check, however, is the required attire. To attain accurate measurements, an ASCAN dresses down to only a pair of thin running shorts, for a man, or a gymnastics-style leotard, for a woman. Surrounded by a suit engineer and any number of suit technicians, an ASCAN must check his or her modesty at the door.

The single most time consuming portion of the first fit check, however, revolves around hand measurements. Working efficiently in the EMU gloves requires dexterity; a poor glove fit will very quickly lead to hand and forearm fatigue, first reducing efficiency and eventually reducing a crewmember's ability to remain safe. Because of the dangers associated with hand fatigue, proper glove fit is paramount. To combat the risks associated with poorly-fitting gloves, the suit engineer gathers 16 separate measurements of each hand. These measurements are then tabulated and compared to the many glove sizes in inventory. Over the years, a number of crewmember specific gloves have been constructed by the EMU manufacturer, ILC Dover, of Dover, Delaware. Since the construction of an entirely new set of gloves is expensive and time consuming (the gloves are constructed from molds made of an individual crewmembers hands), all efforts are made to find a glove style in inventory that can be made to fit a new crewmember's hands. If no sufficient glove can be found, then a crewmember goes through the arduous process of having a pair of gloves built for him or her (Knight, 2009).

At the second fit check session, the focus is all on glove fit. The suit engineer typically provides four viable options that may be resized to fit an individual crewmember's hands. For the next two hours, the crewmember puts on the various glove styles, providing feedback for resizing. The goal is to distribute pressure equally across all fingertips and all finger crotches of each hand. If the glove's 
fingers are too long, there is a loss in dexterity. If the fingers are too short, constant fingertip pressure can cause nerve damage. Wide glove fingers again cause a loss of dexterity, while narrow fingers increase the risk of fingernail delamination.

Using an evacuated glove box, a crewmember is able to wear each set of gloves and manipulate tools while remaining in a shirtsleeve environment and providing constant feedback to improve glove fit. The goal of this session is to find two satisfactory glove styles, the best fitting gloves designated as a crewmember's "prime gloves," and the next best fitting style designated the "backup gloves." Since there only exists one pair of each style of glove in the Class 1 Flight Qualified hardware inventory, each crewmember must possess a set of backup gloves. Should the prime gloves be damaged during an EVA, a crewmember has a set of backup gloves on orbit and available for any subsequent EVAs.

The final fit check session is a dress rehearsal for the Suit Qualification. Upon arrival to the Suit Laboratory, a crewmember dresses in the Liquid Cooling and Ventilation Garment (LCVG) prior to donning the EMU. The LCVG is a full-body garment with narrow diameter lines woven throughout. Once attached to the EMU water supply, the LCVG acts as a heat exchanger, pumping cold water through the lines, collecting and rejecting body heat. On orbit, this heat is rejected through the Primary Life Support System (PLSS), the self-contained backpack worn on the EMU. In the NBL, however, all life support systems are supplied through a surface umbilical connected to the back of the EMU.

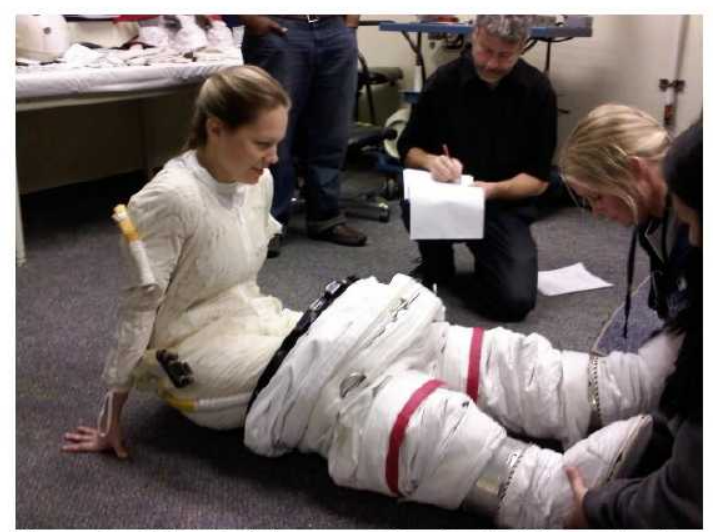

Figure 2. EVA Task instructor Dr. Sandra Moore donning the LTA during the third fit check session. A suit technician assists Dr. Moore by manipulating the LTA's inner bladder, to clear space for Dr. Moore to insert her foot into the boot. Photo contributed from Sandra K. Moore personal archive
Once attired in the LCVG, the crewmember dons the LTA (Figure 2), then ingresses the Hard Upper Torso (HUT), which is mounted to a stanchion. The weight of the HUT requires it be mounted to a stanchion, and the stanchion's height is adjusted to match the height of the crewmember, in the standing position. Universally this is regarded as the most challenging part of donning the EMU. The HUT body seal closure is 16 inches in diameter, but more encumbering is the angle of the shoulder bearings. Slanted from the top of the shoulder outward to the armpit, the shoulder bearings demand flexibility and patience. Rushing through this portion of the donning process could result in severe shoulder injury.

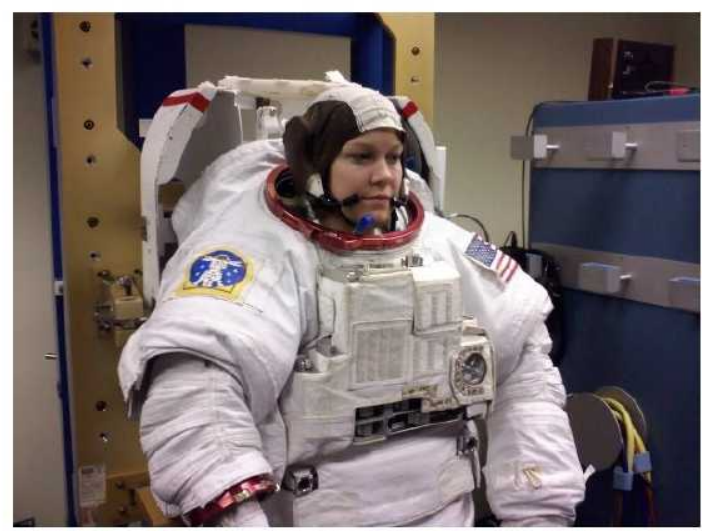

Figure 3. Dr. Moore in the HUT, with the HUT-to-LTA body seal closure locked. Before pressurization can occur, the right glove and helmet will be installed.

Photo contributed from Sandra K. Moore personal archive

Once the HUT is donned, the HUT-toLTA body seal closure is locked and technicians assist the crewmember through the remainder of the donning process. The communications cap, sometimes referred to as the Snoopy Cap, is worn on the head and provides microphones and earphones to facilitate communication (Figure 3). Thin under-gloves are put on for comfort, and then the EMU gloves are donned. Finally, the helmet is lowered over the head and locked in place with a latching neck ring.

With the helmet in place, the EMU is pressurized to $4.0 \mathrm{psid}$ and the suit engineer walks the crewmember through the various activities to evaluate the fit. Arm and leg lengths can be adjusted by rotating cams and inserting or removing expansion rings. The crewmember is also asked to perform a reach evaluation, to determine the extent of his or her reach. When all necessary adjustments have been made, and 
the crewmember and suit engineer decide the suit fit is adequate, the suit is depressurized and the crewmember reverses the series of steps to doff the EMU. The final configuration of the EMU is recorded for future use; due to the limited number of training units, each EMU is built up for a specific crewmember prior to each NBL run.

With the completion of the fit checks, the first prerequisite is complete; the EMU can be sized for the crewmember, ensuring he or she can work in the EMU with an appreciably reduced risk of injury.

\section{NBL SCUBA Qualification}

Many who enter the astronaut corps already hold open-water SCUBA certification, but all are required to complete a qualification course at the NBL. In short, anyone wishing to dive at the NBL must pass a written exam, a swim test and a SCUBA test.

The written exam is similar to an openwater PADI or NAUI certification exam, with the addition of NBL specific NITROX training. A recreational diver uses SCUBA cylinders filled with air, containing $\sim 21 \%$ oxygen. To increase bottom time without appreciably increasing the risk of decompression sickness, the NBL operates using oxygen enriched air. Rather than $\sim 21 \%$ oxygen, the oxygen enriched air used at the NBL is approximately $46 \%$ oxygen. This has the added benefit of reducing the surface interval required between diving and flying. The recreational surface interval between diving and flying is 24 hours. After diving for 3 hours in the NBL on NITROX at 40 feet, however, the surface interval is reduced to just 14 hours. This provides greater scheduling flexibility for the astronauts, whose demands sometimes require flight operations the day following dive operations.

The swim and dive tests evaluate a crewmember's capabilities in the water. Upon the successful completion of these three tests, a crewmember is qualified to SCUBA dive in the NBL and thus perform a Suit Qualification run in the EMU.

\section{EVA-Specific Tools and Hardware Training}

EVA, like NASA itself, is built upon acronyms and nomenclature. As Chris Cassidy, a veteran EVA crewmember who conducted three EVAs on STS-127/2JA in July, 2009, states, there are "lots of new terms to learn as a new EVA crewmember. When you first start out, it's actually difficult to verbalize what you are doing and seeing using the correct terminology." To aid the crewmembers in learning this new language, a series of classes are conducted prior to the Suit Qualification to familiarize the new EVA crewmembers with the tools and hardware. The final class prior to the NBL run is a briefing that covers the various aspects of the Suit Qualification, from the timeline of activities to tips on avoiding some common first-time mistakes.

With these three prerequisites complete EMU fit check, NBL SCUBA qualification and EVA-specific tools and hardware training - an ASCAN is now prepared to take full advantage of the lessons to be learned in the Suit Qualification.

\section{Suit Qualification: The Run}

Like all NBL runs, the Suit Qualification begins on the pool deck at seven a.m. with tool preparation. Each crewmember of the two-person team is tasked with assembling his or her own tools in a preferential configuration. An ASCAN facing his or her first NBL run is at a distinct disadvantage, since he or she has no real preferences as of yet. The ASCANs rely on the EVA Task instructor to provide guidance and a baseline configuration, from which each crewmember can modify at will to meet their needs and preferences.

The crewmembers then go through a routine medical checkup conducted by the flight surgeons on staff at the NBL, and in the locker room, each crewmember dons the LCVG. Following this, the entire test team (including the divers that will be in the water supporting the training, the Test Director in charge of operations at the NBL, the Test Conductor responsible for crew training, the medical staff and the Test Safety Officer) holds a pre-brief to apprise everyone involved of the objectives of the day's training.

The crew returns to the pool deck to don the EMUs, following a protocol similar to that used during the final fit check (Figures 4 and $5)$. 


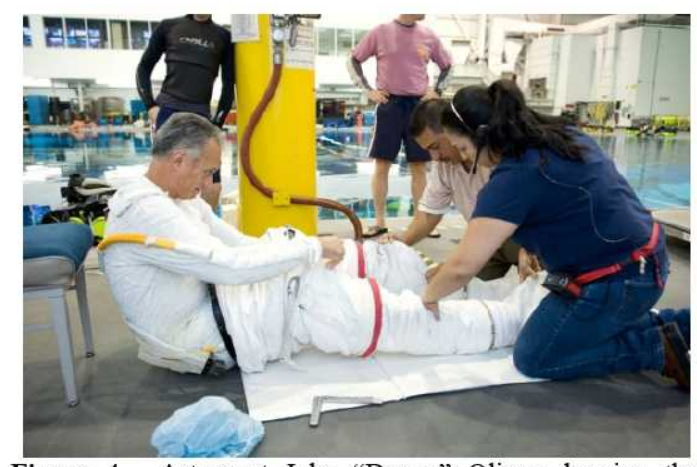

Figure 4. Astronaut John "Danny" Olivas donning the Lower Torso Assembly (LTA) on the NBL pool deck. The "L" shaped handle on the floor to his immediate right is installed on the body seal closure and is used to aid in mating the LTA body seal closure to the Hard Upper Torso (HUT). Source: jsc2009e061367.jpg (photo added 03/25/2009)

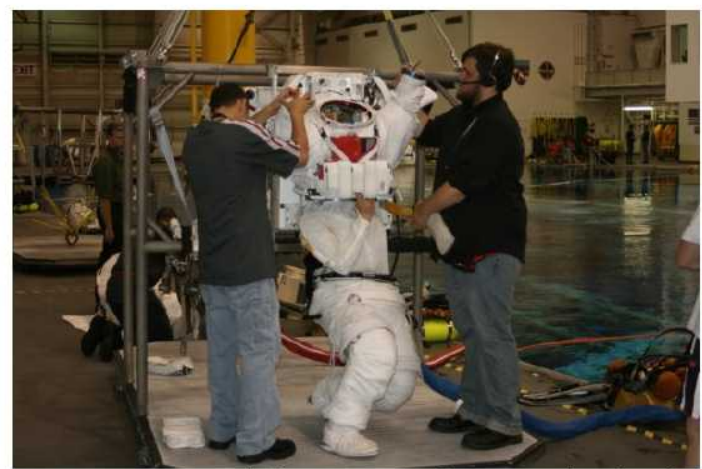

Figure 5. EVA Task instructor Matthew Gast donning the HUT, which is mounted to the lifting platform, prior to a training session in the NBL.

Photo contributed from Matthew Gast personal archive

Once in the HUT with the body seal closure locked, the suit engineer and technicians install the Snoopy Cap, gloves and finally the helmet. Then, slowly, the EMU is pressurized to 4.0 psid. A valsalva device is located in the helmet to help a crewmember clear his or her ears as pressurization takes place. A crewmember simply presses his nose against the valsalva device and gently blows to equalize ear pressure. Since one cannot plug one's nose using a hand when the helmet is installed, the valsalva device is a necessity for most crewmembers. Tool technicians then attach all tools, and members of the dive team attach the leg weights used to help attain the EMU's neutral buoyancy underwater (Figure 6).

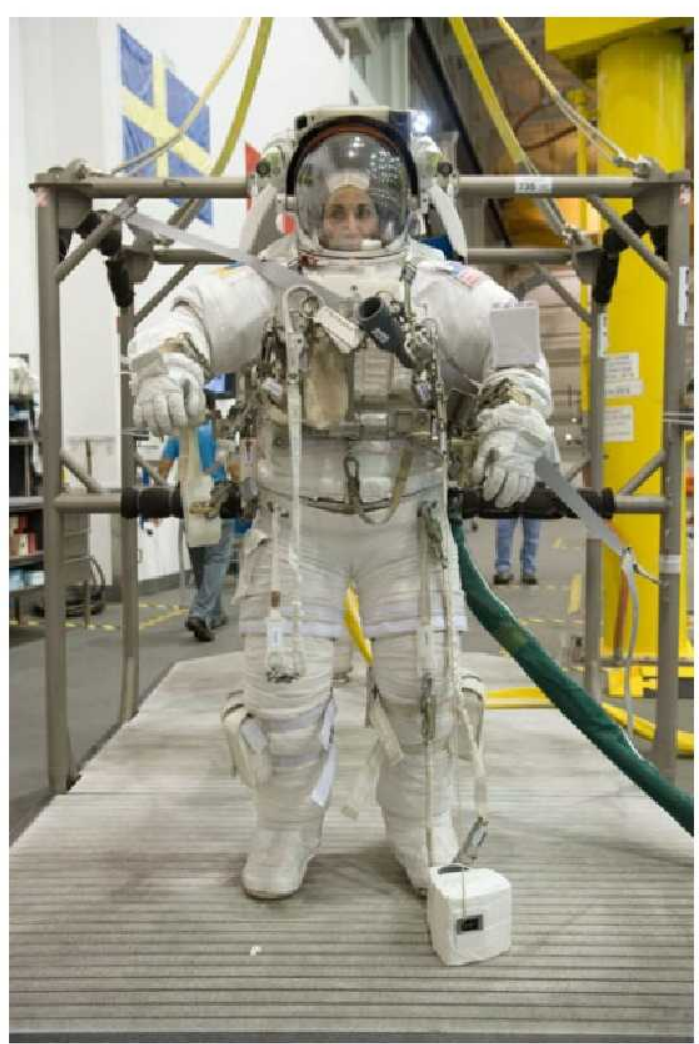

Figure 6. Astronaut Nicole Stott in the sealed EMU at a pressure of $4.0 \mathrm{psid}$, with tools and leg weights installed. Source: jsc2009e070811 (Photo added 04/14/2009)

The last remaining step is to lift the platform into the water using a crane at the water's edge (Figure 7). Once underwater, the NBL Dive Team SCUBA divers release the PLSS from the platform, perform initial buoyancy checks and take the crewmember to a descent line. Since the EMU is not equipped with fins, locomotion in open water is inadvisable. The descent line is a rope routed to the bottom of the pool that a crewmember uses to descend to the pool floor at his own pace, allowing adequate time to equalize ear pressure. 


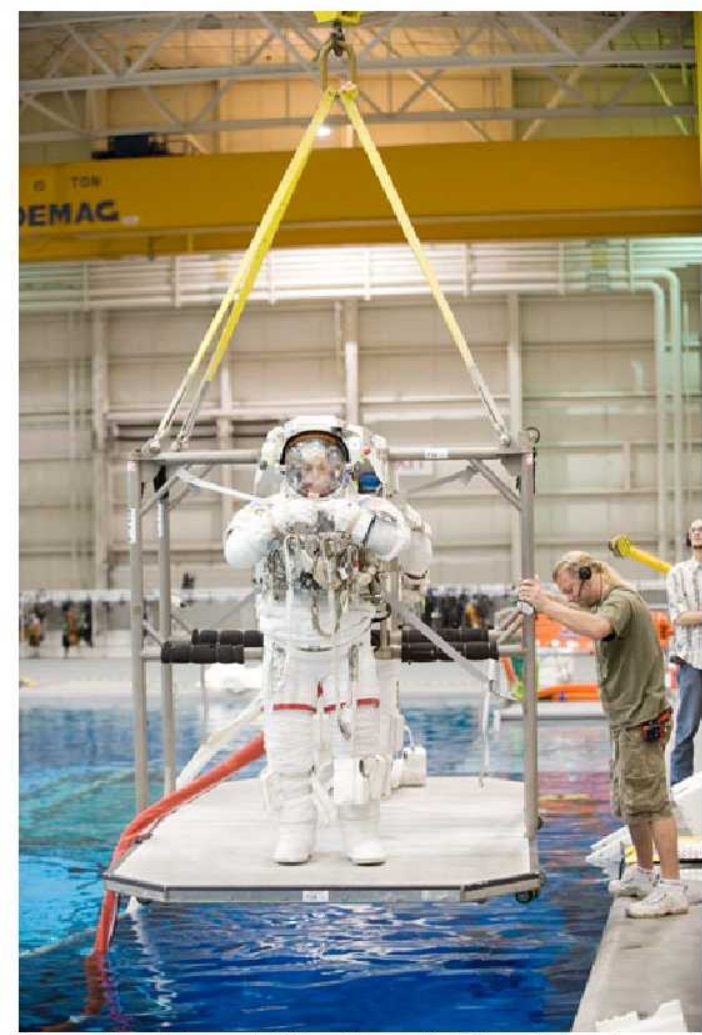

Figure 7. Astronaut John "Danny" Olivas being lowered into the water, to begin a flight-specific run for STS128/17A. ESA astronaut Christer Fuglesang stands on the opposite side of the platform.

Source: jsc2009e061387.jpg (photo added 03/25/2009)

Cassidy described the act of transitioning from air to water this way. "Upon submerging, I experienced 'fish-bowl vision."” The curvature of the helmet visor warps the incoming light, causing a "fish-bowl" effect. Depending on one's susceptibility to motion sickness, the "fish-bowl" effect has been known to cause nausea and disorientation not unlike vertigo. Cassidy continues, "the descent line looked like it was 10 feet away when it was actually within arm's reach. It took my eyes and my mind about 10 minutes to adjust." After that time, as is typical for most crewmembers, the mind adapts to the effect and it is no longer a prominent issue.

At the bottom of the pool, the divers adjust, add, subtract and relocate weight to five different locations on the EMU in an attempt to balance out the weight and buoyancy. The most difficult factor for a new EVA crewmember to discern is a good weigh-out versus a bad one. The divers rely on crewmember input to optimize the weigh-out, but a new EVA crewmember has no basis upon which to offer input, and the divers are left to do their best.
Like many of the other skills learned over the course of training, an EVA crewmember will begin to recognize changes necessary to improve weigh-out, but in this initial stage, they have little insight.

For the next three hours, the crewmembers practice the basics of EVA. They are given eight primary objectives to complete, but those objectives are merely given to introduce the crewmembers to a number of the most important skills. At the top of that list is the ability to translate in the EMU, and the crewmembers will spend the majority of this run, and a preponderance of the following 13 runs, working to improve their ability to translate.

Below are the fundamental objectives for the Suit Qualification. Each of these emphasizes the importance of good translation and body control techniques:

- Translation Adaptation

- Reach Limitations

- Basic EVA Tool Operations

- Articulating Portable Foot Restraint (APFR) Ingress

Wingspan is limited by the shoulder bearings, visibility is limited by the helmet, and brute strength is countered by the inertia of the suit. For these reasons and many others, translating in the EMU is an art. The most basic skill, as stated earlier, is to learn not to fight the suit. Former astronaut Joe Tanner, a spacewalk veteran and EVA mentor, offered the following advice to all new EVA crewmembers: "If you fight the suit, you will lose every time." Much like a boxing match, each blow alone may not be noticeably painful, but the cumulative effect is detrimental to efficiency and safety.

An indicator of efficiency is heat generation. Through the umbilical, the LCVG routes cold water across the entire body, helping to reject body heat. For the NBL, cooling is defined by the number of gallons of cold water pumped through the EMU per hour. On the donning stand after suit-up the typical flow rate is 20 gallons per hour (gph). Due to available resources, the maximum available to any crewmember at any time is 80 gph. A crewmember is responsible for managing his or her own internal environment; a sign that a crewmember is fighting the suit is a request to raise cooling when that crewmember is performing simple tasks. To an EVA Task instructor, this is an indicator that something in the technique being applied needs improvement. 
Beyond the physical aspects, there is little need to add any more to this run to specifically address the mental aspects of EVA; the amount of information being processed each second is mentally taxing in and of itself. It is important, however, to stress just how much multitasking focus a crewmember will need to succeed in the generic training programs. To this end, the Suit Qualification also addresses proper tether protocol.

In microgravity, any hardware not tethered at all times has a significant chance of being lost overboard. For this reason, the ASCAN and Skills Programs repeatedly stress proper tether protocol. Beyond just hardware tethering, however, is safety tethering. It is essential that every EVA crewmember develop an innate ability to manage his or her safety tether (a tether "life line" used to keep a crewmember attached to the ISS). Failure to do so could result in a crewmember losing contact with the ISS and drifting away. It is easy to imagine that having a cord stretched behind you adds complexity to everything you do; it can, and often does, get tangled up in hardware, in the EMU or the PLSS, and even in an EVA crewmate, if vigilance is not maintained. To emphasize the importance of safety tether management, the crewmembers spend the last hour of the Suit Qualification translating around the ISS while attached to safety tethers.

It can seem overwhelming, and the lessons learned during the Suit Qualification are certainly important, but one cannot lose sight of the fact that spacewalking is an exciting adventure, and training for EVA is exciting in its own right. The following is the account of one veteran spacewalking astronaut's Suit Qualification experience. In it, one can sense both the challenges and the pleasures of EVA.

My first time in the EMU was a training event called a "Suit Qual" with a classmate with whom I would one day perform an actual EVA. It was a very low stress affair. Once the helmet was closed and locked, the hardest part of the day was over - or so I thought at the time. We were lowered by crane into the water and once my eyes acclimated to looking through a bowlshaped visor, I was very busy taking in the view of the large space station mock-up sitting in 40 feet of water. We had some simple tasks to perform and before we knew it, we were getting pulled back out of the water - all smiles. The first run was over. As the crane hoisted us out, I began to notice how fatigued I was. I noticed hot spots flaring up on my elbows. My chest was sore on both sides from the bearings that attached the arms to the Hard Upper Torso (HUT). When my gloves were removed, I noticed my fingernails on both my index fingers and thumbs had started to delaminate. After debrief, I went home and took the first afternoon nap I had taken in years. The following morning, I noticed even more reminders of the battle within the suit that had taken place 24 hours prior. The suit had won and I hadn't really realized that I was involved in a fight with it.

All of these physical issues were subsequently resolved through constant tweaking of the suit fit and learning to identify and understand the physical limitations imposed by the suit. Once I understand the limitations, which are frequently task-dependent and therefore an ongoing process, it becomes a game of making adjustments with my technique to minimize further reminders of a day spent in the suit.

It is interesting to reflect on this event that took place a handful of years ago. I remember many of the details as vividly as I remember my first actual EVA.

\section{Suit Qualification: The Glove Pop}

A crewmember can spend three hours in the water in the EMU, practicing translations, tether swaps, foot restraint ingresses, but there still remains one task to accomplish before the training session can officially be termed a Suit Qualification. That one task is the "Glove Pop."

For the safety of the astronauts working in the EMU, it is important for them to experience the most dangerous of all events that could occur in the NBL; that of a rapid EMU depressurization. The training gained from performing a rapid depressurization under ideal conditions, however, negates the risk incurred by intentionally inducing it.

At the completion of the run, the divers return the crewmembers to the donning platform and reattach the PLSS, securing each crewmember to the platform stanchion. Then the Test Director explains the procedure to the EVA crewmember. The diver will place his hands on the wrist bearing of one glove. The Test Director will advise the EVA crewmember to audibly exhale and continue to exhale. While the EVA crewmember is exhaling, the Test Director will direct the diver to release the wrist bearing, detaching the glove from the EMU. Because of 
the pressure differential, the glove will "pop" free of the bearing, but will not come off the crewmember's hand. As one could expect, the crewmember will feel little difference in the HUT, but in the portions of the EMU constructed of soft goods - such as the LTA - the crewmember will feel the EMU constrict. This telltale sign is the indicator that signifies a rapid depressurization. By experiencing it a single time, a crewmember is well prepared to take immediate action, should he or she feel that constriction during a future NBL run.

And because there is positive pressure in the EMU, as long as the crewmember keeps his hand down, little water will enter the suit, even with the glove disconnected. When the glove pop has been performed for both crewmembers, the platform is hoisted from the water and the crewmembers are officially qualified to continue ASCAN training in the NBL.

\section{Life After Suit Qualification}

The day ends with a debrief, where the EVA Task instructor offers constructive feedback on the crewmembers' performance, and the astronauts are given the opportunity to discuss all aspects of the run, and ask any questions that arose from their first experience in the EMU.

As stated above, qualification to continue training is most heavily dependent on the completion of the run, including the glove pop. This may seem counterintuitive, since the EVA Task instructors are on hand to evaluate the astronauts and offer suggestions for improvement. This is due in large part to the fact that the expectations placed upon the astronauts for this first run are simple: be safe and maintain a positive attitude.

"Everyone has a different learning curve," astronaut Jim Dutton accurately explains. "After the four ASCAN runs, I was wondering if it was worth continuing because EVA training had been such a struggle. However, the very next run turned out to be a decisive turning point, and every run after that went well."

Dutton goes on to explain that "I haven't really pinned down what changed in that time - I think it may have been the culmination of multiple things coming together, including finding the right gloves (smaller ones that gave me greater dexterity and less hand fatigue), learning to slow down to speed up, and just getting familiar with the environment."
Each student does have an individual learning curve, and there is insufficient data available after the Suit Qualification to make anything more than a passing initial assessment of anyone's potential to be successful at EVA. For that reason, the instruction team emphasizes being safe, and being positive. $\mathrm{Be}$ it the construction of the ISS or the first step on Mars, EVA is demanding, and if 133 EVAs aboard the ISS has taught the space community one thing, it is that no EVA goes exactly as planned. By maintaining a positive attitude throughout training, crewmembers are better disposed to handle the off-nominal situations that arise during every EVA with grace under fire.

\section{The Purpose of Generic, Skills-Based Training Revisited: A Pilot's Perspective}

Astronaut Barry Wilmore will pilot the space shuttle to the ISS aboard STS-130/20A in the spring of 2010, delivering the final major U.S. component, the Node 3 module Tranquility. The following are his thoughts on generic, skillsbased EVA training. Wilmore successfully completed EVA training in the spring of 2009.

I was selected by NASA to pilot the Space Shuttle, reporting to Johnson Space Center in the Fall of 2000. Historically, Shuttle pilots have not received extensive EVA training, as the risk for those trained to land the Shuttle at the end of the mission was considered too high. Now that the Shuttle era is expected to soon come to an end, that policy is under re-consideration. Fortunately, I found myself available for training during a time when both instructors and facilities were available to support.

I'd been in the Astronaut corps for several years and was familiar with the processes and regimen in place to ensure the safety of EVA crewmembers. However, I wanted to begin training with a "sponge" mindset, and take it all in vice bringing in any preconceived notions of how EVA was done. I expected the instructors to "teach" me the safe, effective and efficient mindset and techniques that would ensure success. That was assuming, of course, that I possessed the required motor skills and cognizance.

The training was challenging and extensive, comparable to many phases of flight training I'd experienced over the years. In flight training you learn that the ability to mentally "stay in 
front of the jet" can pay huge dividends in the successful accomplishment of any task. Likewise, extensive preparation is also vital to ensuring success in the air.

EVA training turned out to be much the same. Tether protocol, tether discipline, situational awareness, body orientation, maneuvering techniques, suit fit, etc. etc. etc.; these are just a brief summary of the requirements that must spin around in one's mind continuously. Staying a few steps ahead of a current task, with the ability to multitask and still think ahead, means the difference between being adequate and being exceptional. A disciplined mindset with respect to each and every task, including those that are agonizingly repetitious, is mandatory. Respect for the suit, and the processes in place to ensure safety, can mean the difference between safely accomplishing a task, or floating off into deep space because of a momentary lack of procedural discipline.

These concepts and many, many more were drilled in to me run after run, and I appreciated it. I now watch my fellow Astronauts on orbit doing amazing things while out EVA, and I realize how much time and dedication throughout literally years of training is required to make it appear simple and easy. Believe me, it's far from easy. Thanks to the dedication of the folks in our EVA training branch and the relentless press and high expectations they place on those they train, we've experienced great success on orbit in the construction of the International Space Station and will do the same as we continue back to the moon and beyond.

\section{Conclusion}

The EVA Suit Qualification is but the first step in a journey that currently leads to the International Space Station, and may soon lead to EVAs on the lunar surface or other terrestrial bodies beyond our small corner of the universe. To face those challenges head on will require continued dedication to generic, skills-based training.

Each success provides more lessons that can be applied to future EVAs. STS-128/17A taught the EVA community that hardware as massive as the ATA can be managed safely by a single crewmember. This feedback expands the defined EVA capabilities and may someday be instrumental in facilitating the successful completion of a task that had been considered too difficult.

For the Increments who will be calling the ISS "home" after the space shuttle retires, there is no clearly defined EVA content. Rather, these citizens of low Earth orbit must be prepared to conduct spacewalks on short notice, to repair or replace hardware that they have, in some cases, not interfaced with in training in years. It is evident in this case as well that success will be dependent on the astronauts' ability to fill in the gaps in their task-specific knowledge with experiences gained through generic training.

Having the confidence to rely on one's fundamental skill-set is at the heart of EVA success. The Suit Qualification run is the first step in gaining both that skill-set, and the confidence to trust it. Once an astronaut possesses both, the limits are boundless.

\section{Acknowledgements}

The authors would like to thank United Space Alliance for their sponsorship to the 2009 IAC conference. Mr. Matthew Gast would like to also thank Dr. Sandra Moore for working with him as co-author. 


\section{References}

1. Wolf, David A, M.D. NASA astronaut. Personal correspondence. 01 July, 2008.

2. Knight, Robert E. EMU suit engineer. Personal correspondence. 14 June, 2009.

3. Cassidy, Christopher J. NASA astronaut. Personal correspondence. 02 September, 2009.

4. Tanner, Joseph R. Former NASA astronaut. Personal correspondence. 12 June, 2008.

5. Anonymous. NASA astronaut. Personal correspondence. August 2009.

6. Dutton, James P. NASA astronaut. Personal correspondence. 30 August 2009.

7. Wilmore, Barry E. NASA astronaut. Personal correspondence. 27 August, 2009.

8. Wedlake, Wayne (repsonsible DX official for Internal DX EVA Tracking History). EVA Task Instructor. August, 2009.

9. NASA PAO via Lisa Shore. "STS-128 / 17A by the numbers" Message to DX EVA Instructors. 14 September. 2000. Email. 